\title{
Effect of Digoxin on A-V Conduction
}

\author{
R. A. J. SPURRELL, A. M. HARRIS, M. R. HOWARD
}

British Medical fournal, 1971, 3, 563-564

TABLE I-Heart Block Complicating Myocardial Infarction

\section{Summary}

The effect of digoxin on $A-V$ conduction in 19 patients with known disease of their conduction tissue was studied while a demand pacing system was in position. Fifteen had transient complete heart block after myocardial infarction and four had chronic intermittent complete heart block. The patients were studied on return to sinus rhythm.

In no instance was a return to either second- or thirddegree heart block precipitated in these patients despite therapeutic levels and, in some cases, high serum level of digoxin for a period of seven days.

The duration of complete heart block after myocardial infarction was 4.3 days in those taking digoxin while in heart block and 3.3 days in those who started digoxin only after return to sinus rhythm.

\section{Introduction}

"The depression of conduction is one of the most definite effects which digitalis produces. Therefore in partial heart block when further interference with the passage of the cardiac impulses from auricles to ventricles is decidedly undesirable, digitalis is contraindicated" (Robinson, 1922). This view is one which is commonly held today, and patients with firstdegree or second-degree heart block who are in congestive heart failure and require digoxin may have this withheld because of the possibility of causing complete heart block. It was therefore decided to study the effect of therapeutic levels of digoxin on $\mathrm{A}-\mathrm{V}$ conduction in order to evaluate its safety in patients with known disease of the conduction tissue.

\section{Patients and Methods}

Nineteen patients ( 15 men and 4 women) were studied15 had transient complete heart block following acute myocardial infarction (Table $\mathrm{I}$ ) and four had chronic intermittent complete heart block, probably due to bilateral bundle-branch fibrosis (Table II). The patients in complete heart block following myocardial infarction were a selected group in that they had been sent from other hospitals for transvenous ventricular pacing, and the study was carried out after the patient had returned to sinus rhythm. Nine patients were digitalized for congestive cardiac failure after the infarction, and the remainder were digitalized only after they had returned to sinus rhythm. The four patients with chronic intermittent complete heart block were studied while they were in sinus rhythm. Throughout the trial of digoxin all patients had a transvenous pacing electrode in the right ventricle attached to a demand pacemaker.

Digoxin $0.5 \mathrm{mg}$ was given eight-hourly for the first day and then digoxin $0.25 \mathrm{mg}$ twice daily (if the blood urea was

Cardiac Department, St. George's Hospital, London S.W.1

R. A. J. SPURRELL, B.SC., M.R.C.P., Registrar (Present address: Cardiac Department, Guy's Hospital, London S.E.1)

A. M. HARRIS, M.D., M.R.C.P., Senior Registrar

M. R. HOWARD, O.M.C., H.M.D., Physics Technician (Present address: Cardiac Department, St. Bartholomew's Hospital, London E.C.1)

\begin{tabular}{|c|c|c|c|c|c|c|}
\hline $\begin{array}{c}\text { Case } \\
\text { No. }\end{array}$ & Progress & $\begin{array}{l}\text { Age and } \\
\text { Sex }\end{array}$ & $\begin{array}{l}\text { Site of } \\
\text { Infarct }\end{array}$ & $\begin{array}{c}\text { Duration } \\
\text { of Heart } \\
\text { Block }\end{array}$ & Digoxin & $\begin{array}{l}\text { Diuretics and } \\
\text { Potassium } \\
\text { Supplements }\end{array}$ \\
\hline $\begin{array}{l}1 \\
2 \\
3 \\
4 \\
5 \\
6 \\
7 \\
8\end{array}$ & $\begin{array}{l}\text { A } \\
\text { A } \\
\text { A } \\
\text { A } \\
\text { A } \\
\text { A } \\
\text { A } \\
\text { D }\end{array}$ & $\begin{array}{ll}52 & M \\
57 & M \\
64 & M \\
47 & M \\
53 & M \\
61 & F \\
55 & M \\
69 & M\end{array}$ & $\begin{array}{l}\text { Inferior } \\
\text { Inferior } \\
\text { Inferior } \\
\text { Inferior } \\
\text { Inferior } \\
\text { Inferior } \\
\text { Inferior } \\
\text { Anterior } \\
\text { and }\end{array}$ & $\begin{array}{l}9 \text { days } \\
2 \text { days } \\
5 \text { days } \\
9 \text { days } \\
4 \text { days } \\
4 \text { days } \\
4 \text { days }\end{array}$ & $\begin{array}{l}\text { On return to S.R. } \\
\text { While in C.H.B. } \\
\text { While in C.H.B. } \\
\text { While in C.H.B. } \\
\text { While in C.H.B. } \\
\text { While in C.H.B. } \\
\text { While in C.H.B. }\end{array}$ & $\begin{array}{l}\text { No } \\
\text { Yes } \\
\text { Yes } \\
\text { Yes } \\
\text { Yes } \\
\text { Yes } \\
\text { Yes }\end{array}$ \\
\hline $\begin{array}{r}9 \\
10 \\
11 \\
12 \\
13 \\
14 \\
15\end{array}$ & $\begin{array}{l}\mathbf{A} \\
\mathbf{A} \\
\mathbf{D} \\
\mathbf{A} \\
\mathbf{A} \\
\mathbf{A} \\
\mathbf{A}\end{array}$ & $\begin{array}{ll}71 & F \\
76 & M \\
49 & M \\
54 & M \\
55 & F \\
64 & M \\
67 & M\end{array}$ & $\begin{array}{l}\text { inferior } \\
\text { Anterior } \\
\text { Anterior } \\
\text { Anterior } \\
\text { Anterior } \\
\text { Anterior } \\
\text { Anterior } \\
\text { Anterior }\end{array}$ & $\begin{array}{l}3 \text { days } \\
3 \text { days } \\
1 \text { day } \\
2 \text { days } \\
3 \text { days } \\
1 \text { day } \\
4 \text { days } \\
6 \text { days }\end{array}$ & $\begin{array}{l}\text { While in C.H.B. } \\
\text { While in C.H.B. } \\
\text { On return to S.R. } \\
\text { On return to S.R. } \\
\text { On return to S.R. } \\
\text { On return to S.R. } \\
\text { On return to S.R. } \\
\text { While in C.H.B. }\end{array}$ & $\begin{array}{l}\text { Yes } \\
\text { Yes } \\
\text { No } \\
\text { Yes } \\
\text { Yes } \\
\text { Yes } \\
\text { Yes } \\
\text { Yes }\end{array}$ \\
\hline
\end{tabular}

TABLE II-Chronic Intermittent Complete Heart Block

\begin{tabular}{c|c|c|c|c|c}
\hline $\begin{array}{c}\text { Case } \\
\text { No. }\end{array}$ & Age & Sex & E.C.G. & Digoxin & $\begin{array}{c}\text { Diuretics and } \\
\text { Potassium } \\
\text { Supplements }\end{array}$ \\
\hline 16 & 75 & $M$ & $\begin{array}{l}\text { R.B.B.B., L.A.D. } \\
\text { Intermittent C.H.B. }\end{array}$ & $\begin{array}{l}\text { On digoxin } \\
\text { when in block } \\
\text { On digoxin } \\
\text { in S.R. only } \\
\text { On digoxin } \\
\text { in S.R. only } \\
\text { On digoxin }\end{array}$ & Yes \\
$\begin{array}{l}\text { R.B.B.B., Normal axis } \\
\text { Intermittent C.H.B. } \\
\text { Axis +70 } \\
\text { Q.R.S. normal } \\
\text { Intermittent C.H.B. } \\
\text { L.B.B.B., L.A.D. } \\
\text { Intermittent C.H.B. }\end{array}$ & 74 & $\mathrm{~F}$ S. only & Yes \\
\hline
\end{tabular}

R.B.B.B. $=$ Right bundle-branch block.

L.A.D. $=$ Left axis deviation.

below $40 \mathrm{mg} / 100 \mathrm{ml}$ ) or digoxin $0.25 \mathrm{mg}$ daily (if the blood urea was above $40 \mathrm{mg} / 100 \mathrm{ml}$ ). Serum digoxin levels were measured daily by a radioimmunoassay technique (Evered et al., 1970) from blood samples drawn before the morning dose of digoxin and the therapeutic level was taken as $1.5 \pm 0.4$ $\mathrm{ng} / \mathrm{ml}$. Lead II of the electrocardiogram was recorded twice daily for seven days for measurement of the P-R interval. Blood urea and potassium levels were measured on two occasions during each study.

\section{Results}

Of the 19 patients studied not one reverted from sinus rhythm to either second-degree or third-degree heart block. Ten had prolonged P-R intervals at the beginning of the study (Cases 1, 2, 4, 5, 8, 9, 10, 15, 18, and 19) (Table III). Four of these (Cases 1, 10, 18, and 19) had had rhythm strips taken before digitalization. The P-R interval shortened in six patients -three (Cases 2, 8, and 10) had high serum levels of digoxin, one (Case 18) had therapeutic levels, and two (Cases 1 and 5) had subtherapeutic levels. The P-R interval was unchanged in two patients (Cases 4 and 15)-one had therapeutic levels and the other high levels of digoxin. The P-R interval lengthened in two patients (Cases 9 and 19)-one had therapeutic levels and the other high levels of digoxin. The remaining nine patients had normal P-R intervals at the beginning of the study and the P-R interval increased in only two of these (Cases 12 and 16). Both patients had high serum levels of digoxin. 
TABLE III $-P-R$ Interval (seconds) and Levels of Digoxin $(\mathrm{ng} / \mathrm{ml})$

\begin{tabular}{|c|c|c|c|c|c|c|c|c|c|c|c|c|c|c|c|c|c|c|c|}
\hline & & & & Befo & Dig. & & & & & & & & & & & & & & \\
\hline & & No. & & $\begin{array}{l}\text { P-R } \\
\text { Int. }\end{array}$ & $\begin{array}{l}\text { Dig. } \\
\text { Level }\end{array}$ & $\begin{array}{l}\text { P-R } \\
\text { Int. }\end{array}$ & $\begin{array}{l}\text { Dig. } \\
\text { Level }\end{array}$ & $\begin{array}{l}\text { P-R } \\
\text { Int. }\end{array}$ & $\begin{array}{l}\text { Dig. } \\
\text { Level }\end{array}$ & $\begin{array}{l}\text { P-R } \\
\text { Int. }\end{array}$ & $\begin{array}{l}\text { Dig. } \\
\text { Level }\end{array}$ & $\begin{array}{l}\text { P-R } \\
\text { Int. }\end{array}$ & $\begin{array}{c}\text { Dig. } \\
\text { Level }\end{array}$ & $\begin{array}{l}\text { P-R } \\
\text { Int. }\end{array}$ & $\begin{array}{c}\text { Dig. } \\
\text { Level }\end{array}$ & $\begin{array}{l}\text { P-R } \\
\text { Int. }\end{array}$ & $\begin{array}{l}\text { Dig. } \\
\text { Level }\end{array}$ & $\begin{array}{l}\text { P-R } \\
\text { Int. }\end{array}$ & $\begin{array}{l}\text { Dig. } \\
\text { Level }\end{array}$ \\
\hline 1 & . & . & . & $\begin{array}{l}0.24 \\
0.24\end{array}$ & $<0.25$ & $\begin{array}{l}0 \cdot 24 \\
0 \cdot 24\end{array}$ & 0.25 & $\begin{array}{l}0 \cdot 24 \\
0 \cdot 24\end{array}$ & 0.5 & $\begin{array}{l}0.22 \\
0.22\end{array}$ & 0.4 & $\begin{array}{l}0 \cdot 22 \\
0.22\end{array}$ & 0.8 & $\begin{array}{l}0 \cdot 22 \\
0.22\end{array}$ & 0.6 & $\begin{array}{l}0.22 \\
0.22\end{array}$ & 0.6 & $\begin{array}{l}0.22 \\
0.22\end{array}$ & $1 \cdot 1$ \\
\hline 2 & . & . & . & - & - & $\begin{array}{l}0.24 \\
0.24\end{array}$ & 3.0 & $\begin{array}{l}0 \cdot 20 \\
0 \cdot 20\end{array}$ & $2 \cdot 6$ & $\begin{array}{l}0 \cdot 20 \\
0.20\end{array}$ & 1.9 & $\begin{array}{l}0.19 \\
0.19\end{array}$ & $2 \cdot 3$ & $\begin{array}{l}0 \cdot 20 \\
0.17\end{array}$ & $3 \cdot 0$ & $\begin{array}{l}0.17 \\
0.20\end{array}$ & 1.3 & $\begin{array}{l}0.18 \\
0.18\end{array}$ & $1 \cdot 1$ \\
\hline 3 & .. & . & . & - & - & $\begin{array}{l}0 \cdot 16 \\
0.16\end{array}$ & - & $\begin{array}{l}0.16 \\
0.16\end{array}$ & 1.8 & $\begin{array}{l}0.16 \\
0.17\end{array}$ & $2 \cdot 0$ & $\begin{array}{l}0.16 \\
0.14\end{array}$ & $2 \cdot 0$ & $\begin{array}{l}0.14 \\
0.16\end{array}$ & $2 \cdot 0$ & $\begin{array}{l}0.16 \\
0.16\end{array}$ & $1 \cdot 8$ & $\begin{array}{l}0.16 \\
0.18\end{array}$ & 1.9 \\
\hline 4 & . & . & $\cdots$ & - & - & $\begin{array}{l}0.24 \\
0.25\end{array}$ & 1.9 & $\begin{array}{l}0.25 \\
0.25\end{array}$ & $1 \cdot 8$ & $\begin{array}{l}0.25 \\
0.25\end{array}$ & 1.5 & $\begin{array}{l}0.26 \\
0.24\end{array}$ & $1 \cdot 3$ & $\begin{array}{l}0 \cdot 24 \\
0 \cdot 24\end{array}$ & 1.5 & $\begin{array}{l}0.24 \\
0.24\end{array}$ & $1 \cdot 3$ & $\begin{array}{l}0.24 \\
0.24\end{array}$ & 1.6 \\
\hline 5 & . & $\cdots$ & $\cdots$ & - & - & $\begin{array}{l}0 \cdot 22 \\
0.22\end{array}$ & 0.4 & $\begin{array}{l}0.23 \\
0.23\end{array}$ & 0.7 & $\begin{array}{l}0.20 \\
0.20\end{array}$ & 0.6 & $\begin{array}{l}0.19 \\
0.19\end{array}$ & 0.25 & $\begin{array}{l}0.22 \\
0.22\end{array}$ & 0.5 & $\begin{array}{l}0.22 \\
0.22\end{array}$ & 0.5 & $\begin{array}{l}0.20 \\
0.20\end{array}$ & 0.5 \\
\hline 6 & . & $\cdots$ & $\cdots$ & - & - & $\begin{array}{l}0 \cdot 20 \\
0 \cdot 20\end{array}$ & & $\begin{array}{l}0 \cdot 20 \\
0 \cdot 20\end{array}$ & & $\begin{array}{l}0.18 \\
0.18\end{array}$ & & $\begin{array}{l}0.20 \\
0.20\end{array}$ & & $\begin{array}{l}0.18 \\
0.18\end{array}$ & & $\begin{array}{l}0.18 \\
0.18\end{array}$ & & $\begin{array}{l}0.18 \\
0.20\end{array}$ & \\
\hline 7 & . & . & $\cdots$ & - & - & $\begin{array}{l}0 \cdot 18 \\
0.18\end{array}$ & 1.0 & $\begin{array}{l}0.18 \\
0.18\end{array}$ & $1 \cdot 0$ & $\begin{array}{l}0.18 \\
0.18\end{array}$ & $1 \cdot 2$ & $\begin{array}{l}0.18 \\
0.18\end{array}$ & 1.4 & $\begin{array}{l}0.18 \\
0.18\end{array}$ & 1.8 & $\begin{array}{l}0.18 \\
0.18\end{array}$ & 1.8 & $\begin{array}{l}0 \cdot 18 \\
0 \cdot 18\end{array}$ & $2 \cdot 2$ \\
\hline 8 & . & . & . & - & - & $\begin{array}{l}0 \cdot 28 \\
0 \cdot 28\end{array}$ & $3 \cdot 3$ & $\begin{array}{l}0 \cdot 20 \\
0 \cdot 26\end{array}$ & 1.9 & $\begin{array}{l}0.20 \\
0.22\end{array}$ & $4 \cdot 1$ & $\begin{array}{l}0.22 \\
0.22\end{array}$ & 4.5 & $\begin{array}{l}0 \cdot 28 \\
0 \cdot 28\end{array}$ & $4 \cdot 1$ & $\begin{array}{l}0.24 \\
0.22\end{array}$ & $4 \cdot 5$ & $\begin{array}{l}0.23 \\
0.23\end{array}$ & $4 \cdot 1$ \\
\hline 9 & .. & . & . & - & - & $\begin{array}{l}0.24 \\
0.24\end{array}$ & 1.4 & $\begin{array}{l}0.24 \\
0 \cdot 24\end{array}$ & $2 \cdot 0$ & $\begin{array}{l}0.24 \\
0.24\end{array}$ & 1.9 & $\begin{array}{l}0 \cdot 24 \\
0.24\end{array}$ & 1.8 & $\begin{array}{l}0 \cdot 24 \\
0 \cdot 24\end{array}$ & $1 \cdot 3$ & $\begin{array}{l}0 \cdot 24 \\
0 \cdot 24\end{array}$ & Insuff. & $\begin{array}{l}0.26 \\
0.26\end{array}$ & 2.4 \\
\hline 10 & . & . & $\cdots$ & $\begin{array}{l}0.22 \\
0.22\end{array}$ & $<0.25$ & $\begin{array}{l}0 \cdot 22 \\
0 \cdot 22\end{array}$ & 1.0 & $\begin{array}{l}0.23 \\
0.33\end{array}$ & $1 \cdot 3$ & 0.17 & $1 \cdot 0$ & $\begin{array}{l}0 \cdot 22 \\
0.19\end{array}$ & 1.5 & $\begin{array}{l}0 \cdot 19 \\
0 \cdot 19\end{array}$ & $2 \cdot 4$ & $\begin{array}{l}0 \cdot 18 \\
0 \cdot 19\end{array}$ & 2.5 & $\begin{array}{l}0.19 \\
0.19\end{array}$ & 2.9 \\
\hline 11 & . & . & $\cdots$ & $\begin{array}{l}0.18 \\
0.18\end{array}$ & - & $\begin{array}{l}0.18 \\
0.15\end{array}$ & 1.6 & $\begin{array}{l}0 \cdot 18 \\
0 \cdot 18\end{array}$ & - & $\begin{array}{l}0 \cdot 18 \\
0 \cdot 18\end{array}$ & - & $\begin{array}{l}0.18 \\
0.18\end{array}$ & 0.9 & $\begin{array}{l}0.18 \\
0.18\end{array}$ & 0.7 & $\begin{array}{l}0.18 \\
0.16\end{array}$ & 0.8 & $\begin{array}{l}0.16 \\
0.18\end{array}$ & 0.9 \\
\hline 12 & . & . & $\cdots$ & $\begin{array}{l}0 \cdot 20 \\
0.20\end{array}$ & $<0.25$ & $\begin{array}{l}0 \cdot 20 \\
0 \cdot 20\end{array}$ & Insuff. & $\begin{array}{l}0 \cdot 20 \\
0 \cdot 20\end{array}$ & Insuff. & 0.20 & 1.7 & $\begin{array}{l}0.21 \\
0.21\end{array}$ & $3 \cdot 2$ & $\begin{array}{l}0 \cdot 21 \\
0 \cdot 21\end{array}$ & $3 \cdot 0$ & $\begin{array}{l}0 \cdot 21 \\
0 \cdot 21\end{array}$ & $2 \cdot 8$ & $\begin{array}{l}0.21 \\
0.21\end{array}$ & $2 \cdot 0$ \\
\hline 13 & . & . & . & $\begin{array}{l}0.16 \\
0.17\end{array}$ & - & $\begin{array}{l}0 \cdot 16 \\
0 \cdot 16\end{array}$ & $2 \cdot 3$ & $\begin{array}{l}0 \cdot 16 \\
0 \cdot 16\end{array}$ & 1.8 & $\begin{array}{l}0.16 \\
0.16\end{array}$ & 1.9 & $\begin{array}{l}0.14 \\
0.15\end{array}$ & 1.8 & $\begin{array}{l}0.14 \\
0.16\end{array}$ & $1 \cdot 8$ & $\begin{array}{l}0 \cdot 14 \\
0 \cdot 15\end{array}$ & 1 & $\begin{array}{l}0.14 \\
0.14\end{array}$ & $1 \cdot 1$ \\
\hline 14 & . & . & $\cdots$ & 0.20 & $<0.25$ & $\begin{array}{l}0 \cdot 18 \\
0.18\end{array}$ & $2 \cdot 2$ & $\begin{array}{l}0 \cdot 18 \\
0 \cdot 18\end{array}$ & $2 \cdot 2$ & 1.8 & 1.5 & $\begin{array}{l}0.18 \\
0.18\end{array}$ & 1.6 & $\begin{array}{l}0 \cdot 18 \\
0 \cdot 18\end{array}$ & - & $\begin{array}{l}0 \cdot 18 \\
0.18\end{array}$ & 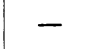 & $\begin{array}{l}0.18 \\
0.19\end{array}$ & - \\
\hline 15 & . & . & . & - & - & $\begin{array}{l}0 \cdot 28 \\
0 \cdot 28\end{array}$ & $2 \cdot 3$ & $\begin{array}{l}0 \cdot 28 \\
0 \cdot 28\end{array}$ & $2 \cdot 4$ & $\begin{array}{l}0.30 \\
0.30\end{array}$ & $3 \cdot 2$ & $\begin{array}{l}0 \cdot 28 \\
0 \cdot 28\end{array}$ & $3 \cdot 8$ & $\begin{array}{l}0 \cdot 28 \\
0 \cdot 28\end{array}$ & 3.6 & $0 \cdot 28$ & - & $\begin{array}{l}0.28 \\
0.28\end{array}$ & 2.9 \\
\hline 16 & . & . & . & - & - & $\begin{array}{l}0 \cdot 16 \\
0.16\end{array}$ & $2 \cdot 3$ & $\begin{array}{l}0 \cdot 18 \\
0 \cdot 18\end{array}$ & $2 \cdot 3$ & $\begin{array}{l}0 \cdot 12 \\
0 \cdot 16\end{array}$ & $3 \cdot 8$ & $\begin{array}{l}0 \cdot 20 \\
0 \cdot 20\end{array}$ & $2 \cdot 7$ & $\begin{array}{l}0 \cdot 20 \\
0 \cdot 20\end{array}$ & $2 \cdot 1$ & $\begin{array}{l}0 \cdot 20 \\
0 \cdot 20\end{array}$ & $3 \cdot 0$ & $\begin{array}{l}0.20 \\
0.20\end{array}$ & Insuff. \\
\hline 17 & . & . & . & $\begin{array}{l}0.18 \\
0.18\end{array}$ & - & $\begin{array}{l}0 \cdot 16 \\
0 \cdot 18\end{array}$ & 1.9 & $\begin{array}{l}0 \cdot 19 \\
0 \cdot 18\end{array}$ & $1 \cdot 8$ & $\begin{array}{l}0 \cdot 19 \\
0 \cdot 19\end{array}$ & 1.7 & $\begin{array}{l}0 \cdot 19 \\
0 \cdot 19\end{array}$ & 1.9 & $\begin{array}{l}0 \cdot 19 \\
0 \cdot 19\end{array}$ & 1.7 & $\begin{array}{l}0 \cdot 16 \\
0 \cdot 16\end{array}$ & $1 \cdot 3$ & $\begin{array}{l}0.16 \\
0.18\end{array}$ & 1.6 \\
\hline 18 & . & . & . & $\begin{array}{l}0.32 \\
0.33\end{array}$ & $<0.25$ & $\begin{array}{l}0.32 \\
0.32\end{array}$ & 1.9 & $\begin{array}{l}0.32 \\
0.33\end{array}$ & $2 \cdot 6$ & $\begin{array}{l}0.33 \\
0.33\end{array}$ & 1.5 & $\begin{array}{l}0.32 \\
0.33\end{array}$ & 1.6 & $\begin{array}{l}0.30 \\
0.31\end{array}$ & 1.5 & 0.31 & 1.6 & $\begin{array}{l}0.30 \\
0.30\end{array}$ & - \\
\hline 19 & .. & . & . & $\begin{array}{l}0.22 \\
0.22\end{array}$ & $<0.25$ & $\begin{array}{l}0 \cdot 22 \\
0 \cdot 22\end{array}$ & 3.0 & $\begin{array}{l}0.22 \\
0.24\end{array}$ & $2 \cdot 3$ & $\begin{array}{l}0 \cdot 24 \\
0.24\end{array}$ & $3 \cdot 0$ & $\begin{array}{l}0 \cdot 22 \\
0 \cdot 24\end{array}$ & 3.7 & $\begin{array}{l}0.26 \\
0.26\end{array}$ & $4 \cdot 0$ & $\begin{array}{l}0 \cdot 26 \\
0 \cdot 26\end{array}$ & 3.4 & $\begin{array}{l}0.26 \\
0.26\end{array}$ & $3 \cdot 1$ \\
\hline
\end{tabular}

Two patients require special mention. One (Case 8) had widely varying $P-R$ intervals, and this was found to be due to intermittent left bundle-branch block, P-R intervals of 0.26 second or over being associated with left bundle-branch block and under 0.26 second being associated with a normal width QRS. The other (Case 10 ) also had varying P-R intervals due to a wandering atrial pacemaker.

The average duration of heart block in the 15 postmyocardial infarct cases was 3.3 days for the six patients not taking digoxin while in complete heart block and 4.3 days for the nine patients taking digoxin.

\section{Discussion}

It is generally accepted that in patients with impaired $A-V$ conduction as shown by a prolonged P-R interval digitalis should be avoided if possible. Blumgart and Altschule (1939) showed that in 19 patients with a prolonged P-R interval due to ischaemic or rheumatic heart disease digitalis could be given in therapeutic doses without precipitating either second-degree or third-degree heart block. We have again studied a group of patients with known disease of their conduction issues and have been able to observe the adequacy of digitalization by measuring serum digoxin levels. We have taken patients with chronic intermittent complete heart block and patients who have had complete heart block following a myocardial infarction and have returned to sinus rhythm. Ideally we would have liked to have studied the effect of digoxin on A-V conduction in patients with a prolonged $\mathrm{P}-\mathrm{R}$ interval following a myocardial infarction, but who had not developed complete heart block. At the time of starting the study, however, it was felt that this was not ethically justified.

Chamberlain et al. (1970) showed that patients with normal renal function on $0.5 \mathrm{mg}$ of digoxin per day had serum digoxin levels of $1.5 \pm 0.4 \mathrm{ng} / \mathrm{ml}$. They also showed that toxic effects of digoxin occurred only at serum levels of $2.0 \mathrm{ng} / \mathrm{ml}$ and over, though patients could have serum levels of $2 \cdot 0-4 \cdot 0 \mathrm{ng} / \mathrm{ml}$ and not necessarily exhibit toxicity. It could be argued that serum levels of digoxin do not give a true measure of its activity in the heart. Doherty et al. (1967) showed that plasma concentrations of digoxin bear enough relationship to those in the myocardium to be of clinical value. Nevertheless, the possibility cannot be excluded that long-term administration of digoxin may have a greater effect on conduction than expected from serum levels, since this study had to be confined to the limited period of seven days when the patient was protected by a demand pacemaker while in hospital.

This study has shown that in patients who are known to have a diseased conduction system it is safe to give therapeutic doses of digoxin for a period up to seven days without precipitating second-degree or third-degree heart block, though there may be minor variations in P-R intervals. Even in the group of patients who had high plasma levels of digoxin seconddegree or third-degree block was not seen. The duration of complete heart block following myocardial infarction seemed to be increased from an average of 3.3 days to 4.3 days if digoxin was being taken while in heart block. No definite statement can be made concerning this as only a few patients were studied. Also the two groups of patients were not comparable with respect to the site of myocardial infarction and the clinical state of the patients. Nevertheless, the average length of time in heart block following myocardial infarction in the last 100 patients at St. George's Hospital was 4.2 days (Harris and Leatham, personal communication, 1970).

We wish to thank Dr. A. G. Leatham for allowing us to study patients under his care, Miss A. Edwards for technical help, and Miss F. Orr for secretarial assistance.

\section{References}

Blumgart, H. L., and Altschule, M. D. (1939). American fournal of Medical Science, 198, 4, 455.

Chamberlain, D. A., White, R. J., Howard, M. R., and Smith, T. W. (1970). British Medical fournal, 3, 429.

Doherty, J. E., Perkins, W. H., and Flanigan, W. J. (1967). Annals of Internal Medicine, 66, 116 .

Evered, D. C., Chapman, C., and Hayter, C. J. (1970). British Medical fournal, 3, 247.

Harris, A. M., and Leatham, A. G. (1970). Personal communication.

Robinson, G. C. (1922). Medicine, 11 . 